\title{
Analysis of Risk Dependencies in Collaborative Risk Management
}

\author{
Catherine de L. Barchet, Luís A. L. Silva, Lisandra M. Fontoura \\ Programa de Pós-Graduação em Ciência da Computação \\ Universidade Federal de Santa Maria - UFSM \\ Santa Maria, Brasil \\ \{catherine.barchet, silva.luisalvaro, lisandramf\}@gmail.com
}

\begin{abstract}
Risk management aims to discuss the probabilities and consequences of risks on the goals of a software project. In such projects, there are dependence relationships between risks, although they are not treated yet by standard risk management practices. This paper is concerned with the analysis of risk dependencies, where these risks are assessed when multiple project stakeholders are involved in the development of collaborative risk debates. Our approach is based on a dialogue game protocol for collaborative risk management. This protocol mediates not only the discussion tasks of risk identification, risk analysis and risk planning, but also the collaborative debate regarding the identification and treatment of dependent risks. These risk management concepts are represented in a Bayesian network model for a risk management discussion situation, where alternative simulation scenarios can be proposed and tested in this probabilistic model according to discussion participants' requests. As observed in a case study, results from this process lead to the enhancement of the argumentative analysis of risk management issues developed by project stakeholders.
\end{abstract}

Keywords - Risk dependencies; Collaborative risk management; Dialogue game protocol; Bayesian networks.

\section{INTRODUCTION}

Software projects have a high probability of failing because their stakeholders are not involved in the development of risk management tasks [1]. Despite this fact, risk management practices are presented in the project management literature - PMBOK (Project Management Body of Knowledge) [2], and in the literature regarding the utilization and evaluation of software development processes - RUP (Rational Unified Process) [3], CMMI (Capacility Maturity Model Integration) [1] and ISO/IEC 15504 [4]. These standard frameworks for risk management, which often rely on experts' judgment of a problem, define risk evaluation tasks where risks are regarded as independent events. However, when dependent risks exist in a software project, a risk may have a significant impact in another risk. The problem is that these risk dependencies are not captured in most risk management frameworks. Important information in the risk management process is being underused or even lost since the explicit analysis of such dependencies can allow project stakeholders to have means of constructing more effective strategies of risk management, reaching better decisions regarding the proposition and analysis of plans to deal with these risks, as observed in [5], for instance. The importance of dealing with risk dependencies is highlighted We gratefully acknowledge financial support from CAPES Brazil. in [6] when stating that the evaluation and mitigation of risks may demand the analysis of complex networks representing risk dependency relationships. According to the CMMI standard [1] and as described in [7], the identification of cause-effect relationships between risks allows a more effective treatment of these risks since the exploitation of these relationships is likely to result in more comprehensive analysis of a risk management problem.

Past work in our research group describes a collaborative approach for risk management in software projects [8][9]. Here, we enhance this approach to deal with the collaborative identification and evaluation of risk dependencies. This is achieved through the expansion of a risk discussion protocol, which is formalized as a "dialogue game" [10] for collaborative risk management. Such kind of protocol amounts to a knowledge acquisition and representation solution for challenges that appear when there is a need of organizing the interchange of arguments that occurs when various project stakeholders collaborate on the deliberation of risk management situation. Based on this argumentation technique, the enhancement of this protocol which is discussed in this paper allows project stakeholders to debate the occurrence and impact of risk dependencies. Then, the information that is captured when these collaborative discussions are developed is utilized on the construction of a Bayesian network - BN [11] model for the assessment of a risk management problem. Through it, project stakeholders can simulate outcomes of a project using a graph representation that contain probability estimates linked to risks, risk causes and risk treatment plans. Our approach is implemented on a new version of a web-based system for collaborative risk discussion - RD System [8][9]. In summary, this work discusses an approach which aims the analysis and simulation of risk dependencies in software projects, where such dependencies are discussed collaboratively in a context where uncertainty is present.

In this paper, Section II discusses argumentation and risk management issues; Section III describes our enhanced dialogue game protocol for collaborative risk management of risk dependencies; Section IV exploits a $\mathrm{BN}$ model in the simulation of risk management issues; Section V discusses a case study carried out in our project and Section VI presents final remarks. 


\section{THE ANALYSIS OF RISK DEPENDENCIES IN COLLABORATIVE RISK MANAGEMENT}

A risk consists on the effect of an uncertainty on the goals of a project [12]. In general, the goals of a risk management process are the identification, evaluation, treatment and minimization of risk items before they turn up as a threat for the successful execution of a project. As described in the CMMI standard [1], to the development of an effective risk management task it is necessary to have a process of risk identification in which project stakeholders are able to collaborate, in which there is a free and open debate of project risks. In addition, the judgment due to the group is likely to act as an aid on the assessment of risks, often promoting higher levels of trust on risk management plans constructed. Collaborative tasks of risk management can be structured by means of argumentation models [13][10], as proposed in [8][9]. According to [10], the modelling and representation of dialogues can be developed when "dialogue game" techniques are exploited in the construction of intelligent systems. A dialogue game [8] is a knowledge representation formalism which recognizes relevant moves of human interaction in debates involving two or more participants. A key task for the representation of a dialogue game is the definition of a set of locutions that can be utilized by debate participants. Each locution captures a participant intention to speak, such as to propose something, to answer a query, etc.

In the process of collaborative risk discussion, participants can identify risks that are dependent on other risks, since they are subject to the effects of other risks. For instance, the increment of the probability of a risk " $\mathrm{A}$ " may influence the probability of a risk " $B$ " to occur in a project, in case the risk " $\mathrm{B}$ " is dependent on the risk " $\mathrm{A}$ ". A deeper level of dependency relationship between risks involves the identification of dependencies that are grounded on risk causes. For instance, risks " $A$ " and "B" can have a common cause, and be dependent because of this. According to CMMI [1], the relationship between risks that are dependent on common causes can make easier the grouping of these risks, leading to risk treatment plans that deal with such common causes. In the analysis of dependent events, it is possible to highlight the exploitation of a BN approach [11], which is a model that permits to handle uncertainty along with the management of risks. This technique is founded on a qualitative analysis, where relevant information for the construction of the $\mathrm{BN}$ model is often obtained when project managers are involved in steps of debate. Most importantly, it is also grounded on the exploitation of quantitative probabilistic analysis which can be tested when a model for a problem situation is constructed. In practice, risk dependency relationships are represented in the $\mathrm{BN}$ model as arcs that link risks represented as nodes of a directed acyclic graph. In situations where risks are dependent because they have causes in common, instead of having risks that are directly linked by dependency arcs, this dependency relationship is represented by a node representing a cause and risks that are dependent on this node. Once this information is modelled, queries can be executed in the $\mathrm{BN}$ graph, which is a task involving the generation of posterior probabilities utilizing probability tables modelled [14] [15].

\section{COLLABORATIVE RISK MANAGEMENT DisCUSSIONS IN THE ANALYSIS OF RISK DEPENDENCIES}

The Risk Discussion (RD) System is a web-based collaborative environment for the elicitation and organization of risk management debates [8][9]. This system mediates the communication between project stakeholders, and consequent standardization of risk information recorded in a project memory. In doing so, the RD System interprets and executes a dialogue game protocol for collaborative risk management [8], which controls the interaction steps between discussion participants according to a set of communication rules. This dialogue game protocol contains a set of locutions that are particularly directed to the capture and representation of typical risk management tasks, namely a) risk identification, b) risk analysis and c) risk planning. Besides of these problem-oriented locutions, the protocol is also formed by general purpose locutions, permitting the full development of multi-participant debates. Among these locutions, for instance, we can cite the "Ask", "Inform", "Argument pro" and "Argument con" locutions (see locutions in [8]). In this paper, we augment this protocol so that project stakeholders can develop dialogues aiming the identification, treatment and simulation of risk dependencies of a risk management problem.

The qualitative analysis of risk dependencies developed when the RD System is used involves the discussion of dependencies between risks which are proposed by debate participants. Once the collaborative identification and analysis of risk dependencies is executed, information related to such dependencies is utilized in the generation of a $\mathrm{BN}$ model. This model allows one to simulate probabilistic predictions of a risk management outcome in a project, using as input the pieces of evidence raised in the debate. This approach is a qualitative and quantitative form of assessing probabilities related to such risk dependencies, and obtaining risk simulation information which can be exploited by project stakeholders in the construction of stronger arguments to be submitted back in a debate. All locutions available in the protocol are relevant to develop risk dependency analysis; although the most exploited locutions are (Fig. 1 presents examples of these locutions):

Locution: propose_risk $(t, P i)$, where $t$ is a description of a risk, and $P i$ is any participant within the dialogue. It permits the statement of risk proposals in a collaborative risk management debate. Preconditions: There must have been utterance of the start_discussion $(t, P i)$ locution by any participant within the dialogue. 


\begin{tabular}{|c|c|c|}
\hline 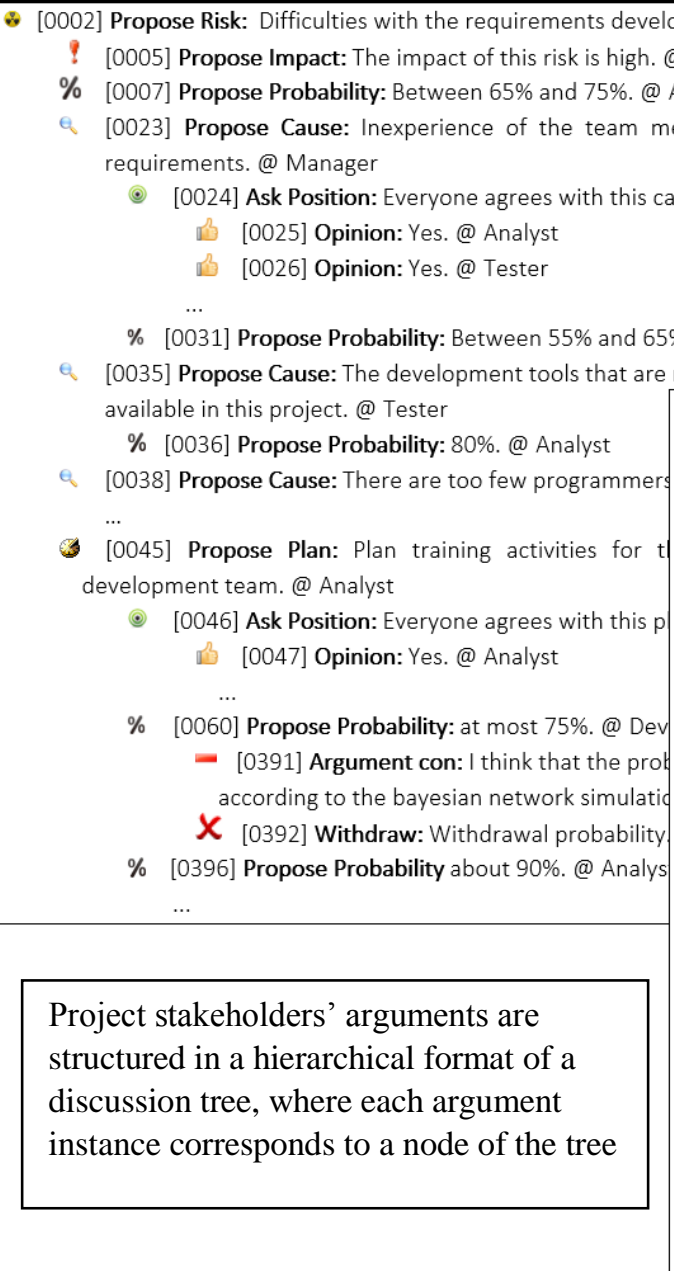 & 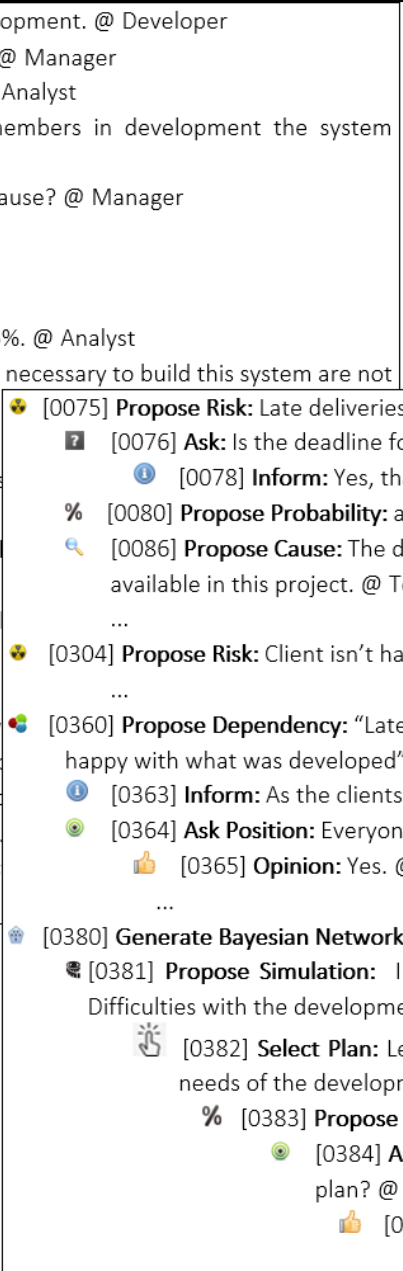 & $\begin{array}{l}\text { the system modules. @ Analyst } \\
\text { developing these modules too close? @Developer } \\
\text { swhy this risk exists. @Manager } \\
\text { nost } 85 \% \text {. @ Developer } \\
\text { elopment tools that are necessary to build this system are not } \\
\text { er } \\
\text { y with what was developed. @ Manager } \\
\text { eliveries of the system modules" is-dependent-on “Client isn't } \\
\text { Manager } \\
\text { y, they are unhappy with these late deliveries. @Manager } \\
\text { grees with this dependency relationship? @ Analyst } \\
\text { ester } \\
\text { he Bayesian Network model was generated. @ System } \\
\text { ink we could reduce still further the effects under the risk } \\
\text { of requirements. @Analyst } \\
\text { select the "Plan training activities for the key programming } \\
\text { nt team" plan. @Manager } \\
\text { obability for State: increase to about } 90 \% \text {. Developer } \\
\text { Position: Everyone agrees with this new probability for this } \\
\text { ster } \\
5] \text { Opinion: Yes. @ Analyst }\end{array}$ \\
\hline
\end{tabular}

Figure 1. A fragment of a collaborative risk debate carried out in the RD System (part of a study case carried out in our project).

Locution: propose_probability $(t, P i)$, where $t$ is a description of a probability estimate, and $P i$ is any participant within the dialogue. Based on project stakeholders' experience, and augmented by discussion in which users can adjust their statements, the use of this locution permits the proposition of probability values regarding the occurrence of risks and/or risk causes. It is also utilized when users state probabilities regarding the effectiveness of risk treatment plans. Similarly, probabilities related to the occurrence of risk causes and the effectiveness of risk plans can also be collected when this locution is used. Preconditions: There must have been utterance of the propose_risk $(t, P i)$, propose_cause $(t, P i)$ or propose_plan $(t$, $P i)$ locutions by any participant within the dialogue.

Locution: propose_plan $(t, P i)$, where $t$ is a description of a risk treatment plan, and $P i$ is any participant within the dialogue. It permits the proposition of plans to treat risks of a software project, where these plans can be directed to the treatment of risks that are dependent of other risks proposed in a debate. Preconditions: There must have been utterance of the propose_risk $(t, P i)$ locution by any participant within the dialogue.
Locution: propose_dependency $(t, P i)$, where $t$ is a statement composed of two risks previously proposed in the debate (along with the reserved word "is-dependenton), and $P i$ is any participant within the dialogue. It permits the identification of binary dependency relationships between two risks of a software project. Important, it is an indexing place for recording of other participants' arguments related to the detailed characterization and analysis of these dependencies. Preconditions: The propose_risk $(t, P i)$ locution should be inserted by any participant at least twice in the dialogue.

Locution: propose_cause $(t, P i)$, where $t$ is the description of a cause of risk, and $P i$ is any participant within the dialogue. It allows participants to indicate possible risk causes in a debate. Preconditions: There must have been utterance of the propose_risk $(t, P i)$ locution by any participant within the dialogue.

Once risk dependencies are made explicit, participants can generate a $\mathrm{BN}$ model for the risk management problem. Having this model, which can be imported by a standard tool for Bayesian analysis - the Netica System [16], discussion participants can evaluate the outcomes of prob- 
abilistic simulations executed on this model. In a debate, these simulation activities are discussed when the following set of locutions are utilized:

Locution: generate_bayesian_network $(t, P i)$, where $t$ is description automatic "The Bayesian Network model was generated", and $P i$ is the RD System. It generates a graph representing the $\mathrm{BN}$ model in which its risk management concepts are captured through the locutions available in the dialogue protocol. This graph model is formed by proposals regarding risks, risk causes, risk plans, and probabilities linked to nodes of the BN model that represent these concepts, in addition to dependency relationships between risks. When this locution is used, the BN model for the risk management concepts available in the latest version of the debate is generated. In this case, an external representation to the RD System of the BN model is produced. Preconditions: There must have been utterance at least two propose_risk $(t, P i)$ locutions by any participant within the dialogue, with proposed_plan $(t, P i)$ and propose_cause $(t, P i)$ locutions associated.

Locution: propose_simulation $(t, P i)$, where $t$ is a description of a simulation desired by any participant, and $P i$ is any participant within the dialogue. It permits the statement of probabilistic simulation scenarios as for promoting the analysis of risks along with their causes, the effectiveness of plans for reducing risks, the effects of risk dependencies, etc. To select risk management concepts to be exploited in a simulation scenario, in which probabilities for variable states can be changed according to users' requests, debate participants can use locutions such as: "Select Plan", "Select Cause" and "Select Risk". To execute this simulation, users state that the probability of a certain plan to be effective should be exploited. This is described through the utilization of "Select Plan" and "Propose Probability for State" locutions, where the first locution selects a plan available in the BN model and the second locution describes that the probability of success of this plan should be changed. Preconditions: There must have been utterance of the generate_bayesian_network $(t, P i)$ locution by any participant within the dialogue.

Locution: select(type, $t, P i)$, where type can be $\{$ risk, cause, plan $\}, t$ is a statement of a risk, cause or plan to be select, and $P i$ is any participant within the dialogue. This locution permits the selection of risk management concepts represented in a BN model. Discussion participants can utilize it when making a detailed discussion of a simulation scenario. In our debate protocol, these simulations are captured and recorded as sub-trees of a "Propose simulation" node. Preconditions: There must have been utterance of the propose_simulation $(t, P i)$ locution by any participant within the dialogue.

Locution: propose_probability_for_state $(t, \quad P i)$, where $t$ is a description of a probability to be simulated in a state of a variable represented in a BN model, and $P i$ is any participant within the dialogue. It permits the proposition of probability estimates for the different states of variables of a BN model. For instance, debate participants can state their beliefs of a risk treatment plan to have success, the belief of a risk cause to be present in a software project, and so on. Such statements are made on the grounds of the pieces of evidence users have observed in the current project or in debates of past projects. Preconditions: There must have been utterance of the select(type, $t, P i)$ locution by any participant within the dialogue.

In addition to the rules that define the set of permitted protocol locutions, rules regulating the combined use of these locutions are represented in this model. These rules allow the RD System to automatically mediate the debate that occurs, regulating the conditions in which certain locutions can be utilized or not. Rule like these are also relevant on the representation of transitions between dialogue phases as, for instance, the transition from a risk identification phase to a risk dependency analysis phase, or a risk dependency simulation phase.

\section{THE EXPLOITATION OF A BAYESIAN NETWORK MODEL IN THE ANALYSIS OF RISK DEPENDENCIES}

The process that leads to the construction of a $\mathrm{BN}$ model representing risks, risk causes, risk treatment plans, and the dependency relationships between these concepts is implemented in the RD System. It is a semi-automatic process in which the nodes and arcs of this Bayesian graph are derived from users' arguments captured when the RD System is used. In essence, users advance these arguments along with the "Propose Risk", "Propose Cause" and "Propose Plan" locutions. Then, this model is imported in the Netica System [16], allowing the participants of a debate to compute with probabilities the outcomes of queries executed in the BN model.

In the $\mathrm{BN}$ model for a problem situation, dependency relationships link risks which were proposed previously in a debate. In the RD System, these relationships can be captured when the "Propose Dependency" locution is used. Risks can also be dependent because they have common causes, as described in [1]. In a debate, these causal dependencies are not captured when users utilize the "Propose Dependency" locutions. To do so, the RD System automatically identifies pairs of risks that have common causes since these statements are captured explicitly when the "Propose Cause" is utilized by debate participants. It is relevant to notice that project stakeholders can exploit these causal dependencies when they plan mitigation actions to take advantage of these dependencies. Once plans are directed to the treatment of risk causes that are relevant to multiple risks, these plans can have a positive effect on these various risks at the same time. To capture these situations, we represent nodes for risk management plans in this $\mathrm{BN}$ model. As shown in Fig. 2, the risk treatment plans proposed in a collaborative risk debate are there in the $\mathrm{BN}$ graph, along with probability estimates regarding the effectiveness of these plans. 


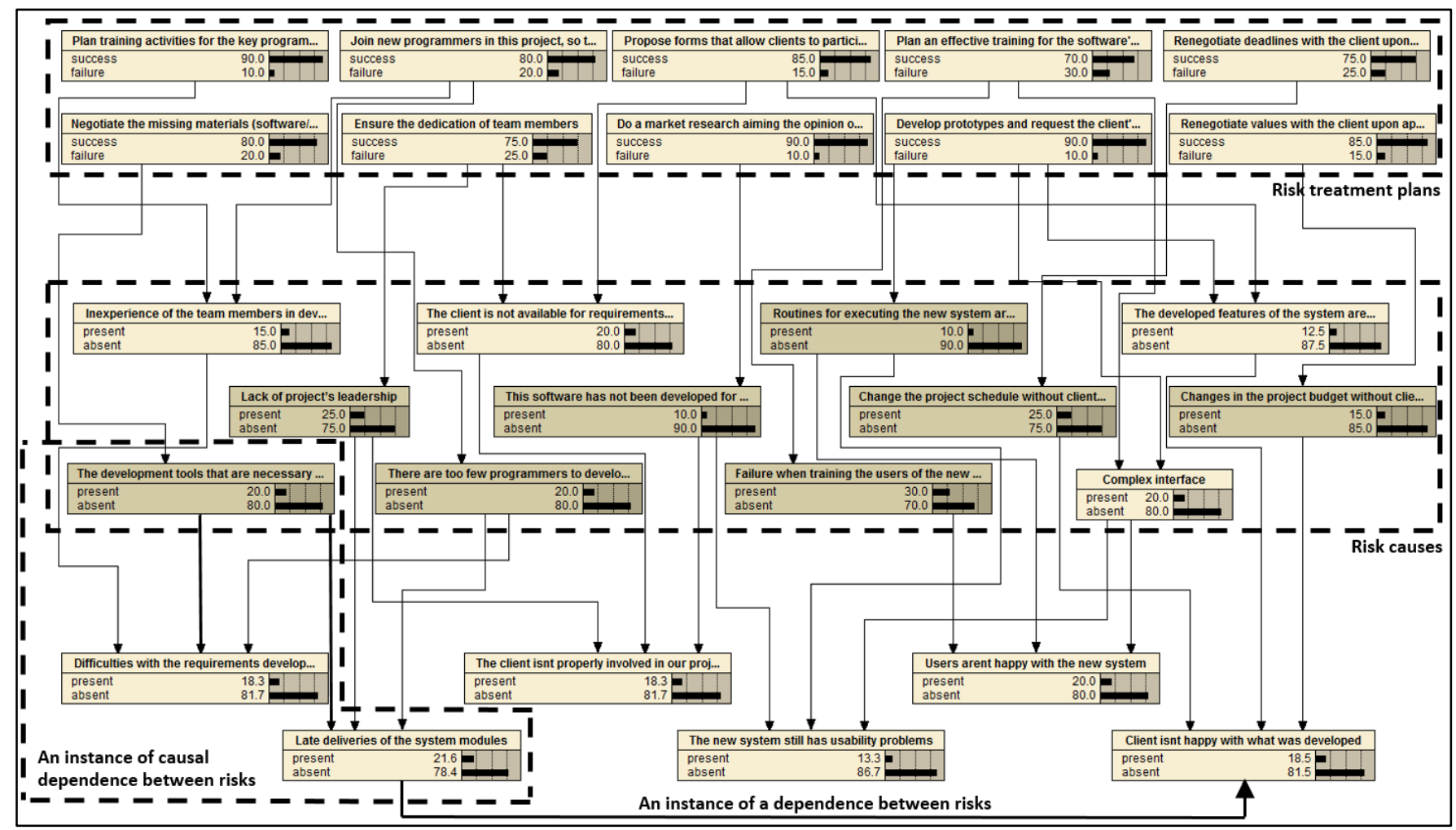

Figure 2. Topology of a Bayesian Network model generated from a collaborative risk debate (part of a study case carried out in our project)

In our BN model, risk management nodes are annotated with binary states (e.g. present and absent states of a risk variable, as in Fig. 2). Then, probability estimates are represented on the binary states of these nodes. In the Netica System, alternative queries can be executed when probability values for these variable states are input (e.g. when users input a certain probability value representing the likelihood of a risk being present in a project). This kind of input can lead to the update of the probability tables connected to the network variables of the $\mathrm{BN}$ model, which is something that the Netica System does automatically. In the assessment of these simulation scenarios, the RD System permits any discussion participant to utilize the "Propose Simulation", "Select Risk", "Select Cause", "Select Plan" and "Propose Probability for State" locutions. Moreover, these issues can be discussed further when other general purpose locutions are utilized (see [8]) - e.g. when users discuss pros and cons of a simulation situation through the utilization of "Argument pro" and "Argument con" locutions. As a result, alternative simulation scenarios can be recorded in a risk management debate (see Fig. 1). For instance, through the combined use of the "Select Risk" and the "Propose Probability for State" locutions, users may state changes on the probability values currently linked to a selected risk. Similarly, changes on the probability values linked to risk causes and risk treatment plans can be stated in such simulation phase of a debate. Once new values of probability are presented by debate users, they can be applied in the probabilistic model which is loaded in the Netica System. This BN model contains variables in which initial probability values are obtained from the participants' statements collected through the use of "Propose Probability" locutions. Other probability values (e.g. posterior probabilities) are related to the outcomes produced when such probabilistic model is computed according to Bayesian rules and probability tables linked to the network variables represented in the BN model. In the simulation phase of a debate, for instance, when users suggest alternative values for probabilities of nodes representing risk treatment plans in the $\mathrm{BN}$ model, this action allows the risk management team to assess the impacts and consequent merits of risk management strategies. In practice, such probability suggestions aim to test different degrees of belief that users may have on the effectiveness of these plans.

\section{REMARKS OF A CASE STUDY}

A preliminary case study developed in our project involved the execution of collaborative tasks of risk identification, risk analysis and risk planning. In addition, a group of participants carried out tasks of identification and analysis of risk dependencies, as well as the simulation of a BN model generated from the risk management concepts proposed in a debate developed in the RD System.

The first task of this case study was the discussion of the risks of a software project. As a result, the $\mathrm{BN}$ model for the current risk management problem was generated through the use of the "Generate Bayesian Network" locution. Then, the resulting probabilistic graph generated by 
the RD System was imported in the Netica System. Using this model, alternative scenarios of simulation suggested were executed. In doing so, participants stated queries based on probability estimates for risks, risk causes and risk treatment plans. To do that, they utilized locutions such as "Propose Simulation", "Select Risk" and "Propose Probability for State", for instance. As a positive feedback from the simulation tasks executed, participants were able to formulate new arguments (e.g. new proposals for risk probability grounded on simulation outcomes) and submit them on the current debate. As an example of this simulations (see Fig. 2), it is possible to observe that the probability of the "Late deliveries of the system modules" risk is $21.6 \%$. This estimate is quite low since the users have a plan to treat the cause of this risk ("The development tools that are necessary to develop this system are not available in this project"), and that the effectiveness of this plan is $80 \%$ as stated by debate participants. As identified collaboratively in the debate, this risk cause is also linked to the "Difficulties with the requirements development" risk, which has probability of $18.3 \%$ (very low as well). This indicates that these two risks have a causal dependency between them and the proposed treatment plan is being applied to this common cause. So, this single plan is managing to deal with two project risks at the same time, which is one of the key advantages of treating risk dependencies in risk management.

\section{CONCLUDING REMARKS}

The collaborative analysis of risk dependencies in software projects is a challenging problem that is tackled superficially in standard risk management frameworks [1][2][3][4]. To approach this problem, this paper discusses the augmentation of a dialogue game protocol for collaborative risk management as presented initially in [8][9]. In general, we show how one can integrate qualitative and quantitative techniques in a collaborative risk management setting. As implemented in a new version of the RD System, this protocol now contains an expanded set of locutions along with additional risk management debate phases, which are directed to the explicit identification and analysis of risk dependencies in a software project. A key feature of this protocol is to offer knowledge acquisition and representation resources to support project stakeholders in the development of debates regarding the occurrence and effects of such risk dependencies.

The proposed approach shows how this debate protocol can guide users on the capture and recording of alternative risk dependency simulation scenarios as they are proposed and adjusted collaboratively in a debate. The debate represented when this protocol is utilized by project stakeholders can be imported in a standard BN system permitting the execution of probabilistic simulations for the investigation of dependent risks. Based on preliminary tests, the outcomes of these simulations reveal possibilities of risk management improvement allowing users to re-estimate identified risks according to risk dependence characteristics. These improvements can be assessed not only by a small group of project managers, as commonly developed in standard risk management frameworks, but also by other project stakeholders through the proposition of new arguments in their collaborative discussions. In fact, the simulations offer feedback for the proposition of new arguments back in the debate, resulting in a qualitative enhancement of the collaborative risk management of a software project.

As future work, we plan to develop new case studies in order to obtain feedback for improving the usability of our approach. We also plan to seek connections between our collaborative risk management approach for the analysis of risk dependencies and logic-based probabilistic argumentation frameworks proposed in the literature [13], as well as making the new version of the RD System available to the public on the web.

\section{REFERENCES}

[1] P. T. CMMI, “CMMI® for Development, Version 1.3," Tech. Rep. C. Carnegie Mellon Univ. Softw. Eng. Inst., no. November, 2010.

[2] I. Project Management Institute, A Guide to the Project Management Body of Knowledge, Fifth Edit. Pennsylvania: Project Management Institute, Inc., 2013.

[3] IBM, "Rational Unified Process (software)." IBM Rational, 2006.

[4] ISO, "ISO/IEC 15504: Information tecnology - Software process assessment," 2005 .

[5] T. W. Kwan and H. K. N. Leung, “A Risk Management Methodology for Project Risk Dependencies," IEEE Trans. Softw. Eng., vol. 37, no. 5, pp. 635-648, Sep. 2011.

[6] T. Alpcan and N. Bambos, "Modeling dependencies in security risk management," 2009 Fourth Int. Conf. Risks Secur. Internet Syst. (CRiSIS 2009), pp. 113-116, Oct. 2009.

[7] T. O. a. Lehtinen, M. V. Mäntylä, J. Vanhanen, J. Itkonen, and C. Lassenius, "Perceived causes of software project failures - An analysis of their relationships," Inf. Softw. Technol., vol. 56, no. 6, pp. 623-643, Jun. 2014

[8] F. S. Severo, L. M. Fontoura, and L. A. L. Silva, "A Dialogue Game Approach to Collaborative Risk Management," Proc. of the 25th Int. Conf. on Software Engineering \& Knowledge Engineering, 2013.

[9] R. C. B. Pozzebon, L. A. L. Silva, and L. Manzoni, "Argumentation Schemes for the Reuse of Argumentation Information in Collaborative Risk Management," in IEEE 15th International Conference on Information Reuse \& Integration (IRI), 2014.

[10]P. Mcburney and S. Parsons, "Dialogue Games for Agent Argumentation," in Argumentation in Artificial Intelligence, G. Simari and I. Rahwan, Eds. Boston, MA: Springer US, 2009, pp. 261-280.

[11] J. Pearl, Probabilistic Reasoning in Intelligent Systems: Networks of Plausible Inference. California, Los Angeles: Morgan Kaufmann, 1988.

[12] ISO, "ISO 31000:2009 - Risk management - Principles and guidelines," 2009.

[13] T. Benchcapon and P. Dunne, "Argumentation in artificial intelligence,” Artif. Intell., vol. 171, no. 10-15, pp. 619-641, Jul. 2007.

[14]C.-F. Fan and Y.-C. Yu, "BBN-based software project risk management," J. Syst. Softw., vol. 73, no. 2, pp. 193-203, Oct. 2004.

[15] Y. Hu, X. Zhang, E. W. T. Ngai, R. Cai, and M. Liu, "Software project risk analysis using Bayesian networks with causality constraints," Decis. Support Syst., vol. 56, pp. 439-449, Dec. 2013.

[16] S. C. Norsys, "Netica Bayesian Belief Network," 1998. [Online]. Available: https://www.norsys.com/. 